\title{
New radiocarbon evidence on early rice consumption and farming in South China
}

Xiaoyan Yang ${ }^{1 *}$, Weiwei Wang ${ }^{1,2}$, Yijie Zhuang ${ }^{3 *}$, Zhao $\mathrm{Li}^{1,2}$, Zhikun $\mathrm{Ma}^{4}$, Yongchao Ma4, Yong $\mathrm{Cui}^{5}$, Jun Wei ${ }^{6}$, Dorian Q. Fuller ${ }^{3}$

${ }^{1}$ Key Lab. of Land Surface Pattern and Simulation, Institute of Geographical Sciences and Natural Resources Research, Chinese Academy of Sciences, Beijing 100101, China

${ }^{2}$ University of Chinese Academy of Sciences, Beijing 100049, China

${ }^{3}$ Institute of Archaeology, University College London, London WC1H OPY, UK

${ }^{4}$ School of Archaeology and Museology, Peking University, Beijing 100871, China

${ }^{5}$ Guangdong Provincial Institute of Cultural Relics and Archaeology, Guangzhou510075, China

${ }^{6}$ Guangdong Museum, Guangzhou 510623, China

* To whom correspondence should be addressed. Email: yangxy@igsnrr.ac.cn and y.zhuang@ucl.ac.uk

\begin{abstract}
While the possibility of indigenous rice cultivation cannot be entirely ruled out, there is increasing evidence suggesting that rice farming was introduced to South China during the late-Holocene. However, determination of the exact timing of the spread of rice farming to South China is fraught with the lack of reliable radiocarbon dates. In this article, we present 15 new AMS 14C dates of charred plant remains recovered from late-Holocene sites of Shixia and Guye in Guangdong Province of South China. Our new AMS 14C dates suggest a later arrival of rice farming in the Pearl River Delta than previously thought. These new AMS 14C dates will shed new lights to an improved understanding of the environmental background and ecology of the southward spread of rice farming.
\end{abstract}

\section{Keywords}


Guangdong, Guye site, late-Holocene, Lingnan Region, Shixia site, southward spread of rice farming

\section{Introduction}

Recent research has suggested that there was a period of intensifying exploitation and possible cultivation of tuber, sago, and other tropical plants during the late-Holocene in South China (Yang et al., 2013; Zhao, 2011), benefiting from the rich ecological diversity in this region. This crucial subsistence development predated and, arguably, paved the way to the beginning of rice farming in the region as the local populations would have become familiar with managing plants and their habitats. Despite its profound influences on local economic and cultural history, timing of the beginning of rice farming in South China remains unclear. On the one hand, archaeological, archaeo-genetic and archaeobotanical research and computational modelling continue to support the southward dispersal of rice agriculture from the Lower Yangtze River and its relationship with the expansion of Austroasiatic, Austro-Thai and Austronesian-speaking populations into Mainland and Island Southeast Asia (e.g. Bellwood, 2005; Castillo et al., 2016; Civan et al., 2015; Diamond and Bellwood, 2003; Fuller, 2011; Higham, 2002; Higham and Lu, 1998; Silva et al., 2015). In contrast, some geneticists have argued that Guangdong should be the core area for initial rice domestication (Huang et al., 2012). Some archaeologists have previously suggested that rice agriculture could have started in South China (Guangdong or Guangxi) by 6000-5500 BP (e.g. Fuller et al., 2011; Lu, 2009; Zhao et al., 2005). More recently, archaeological evidence, however - including human skeletal morphology and ceramic typology, from South China (including Fujian, Guangdong and Guangxi Provinces), Vietnam and Thailand - suggests rice farming immigrants made major cultural and demographic impacts throughout out this region between 5000 and 4000 years ago (Bellwood and Oxenham, 2008; Matsumura and Oxenham, 2014; Oxenham and Matsumura, 2016; Rispoli, 2007; Zhang and Hung, 2010). Nevertheless, the scarcity of archaeobotanical data (especially macro-plant remains derived from systematic examination) in the southern provinces of China has made it difficult to rule out entirely the possibility of indigenous experiments of rice farming before domesticated rice was introduced to the region. There is thus an urgency to re-examine Neolithic 
sequences at key sites in order to understand the precise chronology of early rice farming in South China and its place in Neolithic human ecology.

The Shixia site is located in the hilly area of northern Guangdong Province (Figure 1). Considered as the type site for the Shixia culture, it contains rich Neolithic and early Bronze Age cultural deposits and rice remains. The former includes many archaeological features and abundant polished stone tools and pottery, indicative of farming and sedentism (Guangdong Provincial Institute of Cultural Relics and Archaeology et al., 2014). While several $14 \mathrm{C}$ dates of charcoal have placed the Shixia culture phase I and II occupation at the site to between 5000 and 4500 BP (Guangdong Provincial Institute of Cultural Relics and Archaeology et al., 2014), there are so far no direct 14 C dates for the Shixia culture phase III occupation nor for the rice remains discovered here, making it difficult to ascertain a full chronology of the Shixia culture and the timing of the beginning of rice farming in this region. Located further south in the Pearl River Delta (Figure 1), Guye is another important Neolithic site that has been recently excavated. Not only were abundant archaeological features and artefacts discovered, tens of charred rice seeds were also found during the excavation, making it an important site to examine timing of early rice consumption and farming in the region. Although there is suggestion that rice agriculture existed at Guye by 5000 BP (cf. Zhang and Hung, 2010), there are no direct $14 \mathrm{C}$ dates to support this yet. As a whole, very few direct radiocarbon dates are available from the late-Holocene sites in South China, and the timing of the beginning and/or spread of rice farming from the place where it was originated to South China is highly debated (Zhang and Hung, 2010). For example, using computational modelling based on the collection of a large data, Silva et al. (2015) predict the arrival time of rice farming to South China during the late-Holocene at around 5000 BP. Contrary to this early date suggested by Silva et al. (2015), Zong et al. (2013), in their extensive coring survey of palaeo-environmental and ecological conditions during the lateHolocene in the northern Pearl River deltaic plain, conclude that thanks to the rich natural resources, the Neolithic communities in this region did not develop 'labour-intensive ricebased' agriculture. It was not until 2500-2200 BP that the introduced rice farming took off because of population growth. Similarly Hu et al. (2013), based on sedimentary proxies for Pearl River erosion in ocean sediments, conclude that intensive rice agriculture only became widely established ca. 2500 BP. The above discrepancies between claims for earlier or later rice cultivation and between small-scale early cultivation and intensive, widespread 
agriculture highlight the importance of obtaining more radiocarbon dates on the timing of the beginning and/or spread of rice farming in South China, and more datasets for inferring the importance of rice in South China.

Here, we report new radiocarbon dates from the Guye and Shixia sites in Guangdong Province. These direct AMS $14 \mathrm{C}$ dates derived from charcoal and carbonized seeds of annual or perennial plants, especially those of charred rice grains, provide crucial information regarding the beginning of rice consumption and potentially rice farming at some key sites in South China. These new dates will enable a more robust examination of the archaeobotanical assemblages to address critical issues such as the process of increasing rice consumption and how the newly established rice farming would have adapted to local environments in South China.

\section{Materials and sites}

\section{Guye and Shixia sites in South China}

Guye $\left(22^{\circ} 49.2^{\prime} \mathrm{N}, 112^{\circ} 40.2^{\prime} \mathrm{E}\right)$ is a typical Neolithic shell midden site in the Pearl River Delta of southern subtropical China (Figure 1). The foothills where the site is located were later covered by a paddy field of less than $2.5 \mathrm{~m}$ a.s.l. This creates a waterlogged environment conducive to the preservation of organic remains. $1100 \mathrm{~m} 2$ of the site were revealed during the excavation in 2006. The Neolithic deposits were divided into four layers (layers 4-7), in which abundant potsherds and stone tools were found. The latter include axe, adz, chisel, flapper and grinding tools. More than 30 carbonized rice seeds were recovered from this waterlogged environment along with the acorns, Chinese olives and other plant remains. Shixia $\left(24^{\circ} 6.5^{\prime} \mathrm{N}, 113^{\circ} 5.5^{\prime} \mathrm{E}\right)$ is located on a higher up environment, around $60 \mathrm{~m}$ a.s.l. in northern Guangdong Province. Three excavation seasons were carried out in 1970s and one was conducted in 1985. The cultural deposits were divided into four phases (Guangdong Provincial Institute of Cultural Relics and Archaeology et al., 2014): the pre-Shixia culture layer, the Shixia culture layer, the Shixia III layer and the Bronze Age layer. The Shixia culture layer is further divided into three sub-phases: early, middle and late Shixia cultural phases. A large quantity of rice grains and stalks were recovered from burials, house foundations, pits and hearths of the Shixia cultural layers. Three 14C dates were obtained from charcoal recovered in burial M79 of the early Shixia cultural phase, and burials M26 and M43 of the Middle Shixia cultural phase (Table 1). 


\section{Samples for AMS $14 C$ dating}

For the Guye site, four soot samples adhering to the exterior of the ceramics, 11 carbonized seeds of Chinese olive and acorns, and three rice seeds were dated in the Radiocarbon Dating Laboratory at Peking University, Beijing (Table 2). These three rice seeds (Figure 2(c)) with a higher degree of carbonization among the total 30 rice seeds recovered were from layers $5-7$, respectively.

We checked all unearthed remains from the Shixia site that are stored at the Shixia Museum. One charred rice seed inside a piece of burned clay from the burial M21 belonging to the late Shixia cultural phase was found (Figure 2(d)).The rice seed is preserved well as an intact shape, and the original length and width of the rice are 4.3 and $2.6 \mathrm{~mm}$, respectively, but it was broken unfortunately from the middle part by accident when images were taken. Both sides are slightly flattened and the surface veins are clear. The embryo is lateral and about $1 / 3$ of the whole. The grain was classified as domesticated rice, Oryza sativa subspecies japonica based on previously established criteria for distinguishing common wild rice (Oryza rufipogon sensu lato) and domesticated rice, among both modern comparative material (Castillo et al., 2016; Pang et al., 1995; Wang et al., 1996) and archaeological carbonized material (Castillo et al., 2016; Deng et al., 2015; Fuller et al., 2010). The charred rice was then sent to the Beta Analytic Radiocarbon Dating Laboratory for AMS dating (Table 2).

\section{Results and discussion}

\section{Timing of the beginning of rice consumption and rice farming}

According to these new AMS $14 \mathrm{C}$ dates, the occupation at the Guye site started at around 5500 BP (Figure 3). The rice seeds from layers 5-7 at Guye were in fact later intrusion as their dates fall into later historical periods (Table 2), whereas the date of the charred rice seed from the Shixia site is of the late-Neolithic age (Table 2).

The late-Holocene sites in South China that are key to understand the beginning of rice consumption and farming are primarily located in the Lingnan Region and the mountainous area of Fujian Province. The former includes the Shixia site in northern Guangdong, the Guangxinhe site in western Guangdong, the Guye site in the Pearl River Delta and the Shaxia site in Hong Kong, while the latter includes the Nanshan site and the Tanshishan site, Fujian 
Province (Figure 1; also see Zhang and Hung, 2010 for more detailed information of the sites). Systematic archaeobotanical research is still rare among these sites, and prehistoric rice remains are scattered discoveries during excavations.

The rice remains at the Jiuwuhoushan site were found from firing places and in pottery vessels along with many rice husk impressions left in chunks of burned earth. Microscopic husk patterns suggested that they were of an ancient rice cultivar (Zhang et al., 2008), but what species or subspecies this rice belonged to and how old were these rice remains are still unclear. Other important discoveries include the rice from the Tanshishan site in Fujian and many late-Neolithic sites in coastal Taiwan (Zhang and Hung, 2010). These sites were either excavated in earlier years before systematic flotation was developed or have been completely destroyed, hindering the re-examination of the chronology and ecology of these early rice remains in South China. Our new dates therefore provide an opportunity for an improved understanding of the timing of the beginning of rice consumption and rice farming in South China.

While previous research suggests an early introduction of rice farming to South China (around $5000 \mathrm{BP}$ ), our new AMS $14 \mathrm{C}$ dates indicate a much more complicated picture. The new dates at Guye point to a much later (historical) date of the rice remains, and highlight the important potential problem of intrusive charred seeds from the 'Neolithic' contexts. The importance of direct dating of crop remains to establish antiquity has been highlighted in other parts of the world, where intrusive carbonized grains are not uncommon on Neolithic sites (e.g. Legge, 1986; Stevens and Fuller, 2012; Whitehouse et al., 2014). In the present context, we have no direct chronological evidence of early rice farming at the Guye site, where instead archaeobotanical finds indicate use of wild food plants including Canarium nuts

The AMS 14C date derived directly from charred rice seed from Shixia is between 4300 and $4100 \mathrm{BP}$. Although there was only a single grain, its measurements $(4.3 \times 2.6 \mathrm{~mm})$ fall near the model values of established domesticated rice assemblages from the Yangtze valley, including Baligang during Qujialing and Shijiahe periods (3000-2100 BC) (Deng et al., 2015) and Songze and Liangzhu period assemblages of the Lower Yangtze (3800-2300 BC) (Fuller et al., 2010). It is also comparable to prehistoric domesticated rice grains from Thailand (Castillo et al., 2016), and it can therefore be referred to Oryza sativa subspecies japonica. Contemporary sites in Guangdong, including Guangxinhe, Niling, Chuangbanyang and 
Xiajiaolong (Figure 1), located in the nearby hilly environment share a similar artefact assemblage with that of the Shixia culture, including a wide variety of cooking vessels (Li, 2011) and some charred rice remains (Xiang, 2005, no direct dates), thus indicative of a similar subsistence strategy. For instance, one rice seed was discovered at the Shaxia site in Hong Kong (ca. 4500 BP), but this has not been directly radiocarbon dated (Lu et al., 2005). Although further evidence is required, it appears that rice was commonly consumed in this hilly area of Guangdong during the Shixia Culture in around 5000-4100 BP.

It is possible to provide a Bayesian model of the advent of domesticated rice by assuming the date from Guye represents late forager traditions and the Shixia culture sequence represents the advent of rice cultivation (Figure 3). Using the Bayesian sequence model provided by the OxCal program, v. 3.10 (Bronk, 2005), we find that the transition to the Shixia Rice farming period took place between 3090 and 2730 cal. BC (95.4\% probability). The new direct AMS dates confirm that rice was cultivated by the end of the Shixia period (2340-2130 cal. BC, 95.4\% probability), while our working hypothesis is that rice cultivation was introduced in the early Third Millennium BC with the start of the Shixia culture.

Further dating evidence is needed to establish the exact timing of the southward spread of rice farming to the Pearl River Delta, but earlier economic importance of rice than the Shixia period is unlikely. Previous research on micro-plant remains at the coastal site of Xincun (5300-4500 BP) revealed the importance of palms, roots and tubers in the local plant food exploitation strategy (Yang et al., 2013). This involved management of southern subtropical plants. The case at Xincun suggests that prior to the arrival of rice and rice farming, there was a stage of intensive use of local wild plant foods. The introduction of rice to the region witnessed a mixture of the cultivation of new cultivars with the continuous gathering of wild plant foods in local subsistence. Even after the beginning of rice farming and perhaps other introduced cultivars, wild food gathering could have remained very important.

\section{Local subsistence prior to the beginning of rice farming}

Another important aspect that cannot be entirely ruled out, according to current archaeobotanical data, is the existence of farming experiments of sago and other tropical plants and possibly some ancient rice species before the arrival of new domesticated rice from the Yangtze River. As revealed by recent research on surviving micro-fossil plant remains (e.g. starch grains and phytoliths), in southern subtropical China, there was a period when 'sago-type palms' were predominantly consumed, along with many other subtropical 
plants such as bananas, freshwater roots and tubers and so forth (Yang et al., 2013). Wild rice was probably also eaten, as it is present as minor component in phytolith assemblages. This is likely exploited from wild populations which are likely to have always been extensive in this region (Fuller et al., 2010). It is probable that the local people were consuming many locally available plant foods during the Neolithic period, which sometimes included wild rice prior to the introduction of domesticated rice during the Shixia period. It is also likely that sago, vegecultural foods and wild nuts continued to be consumed alongside rice.

\section{Conclusion}

In this article, we present new AMS $14 \mathrm{C}$ dates on charred plant remains from important sites in South China. Based on these new dates and cross-checked with published dates, we propose a later timing for the arrival of rice farming in the Pearl River Delta in South China than what has sometimes been proposed. This comes close to the modelled arrival age for a diffusion of rice from the Middle or Lower Yangtze based on the Asian Rice Archaeological Database conducted by Silva et al. (2015). Their model 'postulates two independent origins (of rice domestication) in the Yangtze basin' and helps to further articulate an emerging scenario of rice dispersal: a very slow start for the spread of rice because of the prolonged process of its domestication, but 'quickly pick up pace once rice nears full domestication' (Silva et al., 2015). Our model of dates of late foragers at Guye and rice cultivators at Shixia supports a hypothesis that domesticated rice was introduced to the Pearl River Delta region $5000-4700$ years ago.

The two independent domestication centres suggested by Silva et al. and the late arrival of rice in South China contradict the hypothesis of Huang et al. (2012) that rice was domesticated only in the Pearl River Delta. It thus highlights the importance of archaeobotanical evidence for understanding the history of rice; just using modern genetic data on its own is inadequate (Fuller, 2011). Currently, evidence for the cultivation ecology of this early rice in the Pearl River Delta is obscure. Phytolith assemblages and weed seeds from sites in the Yangtze basin indicate that early rice farming was carried out in wet field systems (Deng et al., 2015; Fuller and Qin, 2009; Weisskopf et al., 2015). By contrast, it is postulated that earliest rice farming systems in Southeast Asia were dry or upland systems based only on rainfall and less labourintensive methods (Fuller et al., 2011; Fuller and Qin, 2009). If early rice cultivation was similarly low-intensity dry cultivation in the Guangdong 
region, it might account for the apparent discrepancy with our evidence for the introduction of rice $5000-4300$ years ago and the palaeoecological datasets that recognize the landscape impact of rice agriculture only about 2500 years ago (Hu et al., 2013; Zong et al., 2013), which might record the intensification for rice cultivation methods. Further research is needed on this topic.

While our new AMS $14 C$ dates do suggest a later arrival of rice farming in the Pearl River Delta in South China than sometimes postulated, the sample size is admittedly small. Coupled with the fact that there is a lack of systematic surveys of the late-Holocene sites that are presumably located in a wide variety of environments, it is possible that the timing and routes of the introduction of rice farming to South China are more complicated than currently known and 'the dispersal of rice agriculture was thus not a single event' (Zhang and Hung, 2010). We do not rule out the possibility of earlier arrival of rice farming to this region, or local experiments in rice cultivation prior to the introduction of domesticated rice from the north. The ecological diversity at these late- Neolithic sites and the development of local subsistence packages including the possible cultivation of other plant species, including indigenous vegecultural tree or tuber crops, do not contradict to our conclusions here about the arrival of domesticated Oryza sativa subsp. japonica from the Yangtze region. Our new AMS 14C dates and archaeobotanical research at the Guye site highlight the importance of more archaeobotanical sampling and direct radiocarbon dating of early crop remains in the Pearl River Delta. Our results highlight the importance of more archaeobotanical sampling and direct radiocarbon dating of early crop remains.

\section{Funding}

Funding for this research was provided by the National Natural Science Foundation of China (Grant No. 41371217 and 41230104) and the National Science and Technology Major Project of China (2015CB953801). Research by DQF on early rice cultivation is supported by grant from the UK Natural Environment Research Council (NE/N010957/1).

\section{References}

Bellwood P (2005) First Farmers: The origin of agricultural societies. Blackwell Publishing, Oxford.

Bronk RC (2005) OxCal Program v3. 10. Radiocarbon Accelerator Unit, University of Oxford. 
Civan P, Craig H, Cox CJ et al. (2015) Three geographically separate domestications of Asian rice. Nature Plants 1: 15164

Diamond J and Bellwood P (2003) Farmers and their languages: the first expansions. Science 300 : 597-603.

Fuller DQ (2011) Pathways to Asian Civilizations: Tracing the Origins and Spread of Rice and Rice Cultures. Rice 4:78-92.

Guangdong Provincial Institute of Cultural Relics and Archaeology and Guangdong Museum and Qujiang Museum (2014) Shixia site--Report of the Excavation during 1973-1978.Cutural Relics Press, Beijing (in Chinese).

Higham C and Lu L (1998) The origins and dispersal of rice cultivation: Rice domestication. Antiquity 72:867-877.

Higham C (2002) Early cultures of mainland Southeast Asia. Chicago: Art Media Resources. Huang X, Kurata N, Wei X et al. (2012) A map of rice genome variation reveals the origin of cultivated rice. Nature $490: 497-501$.

Huang X, Yang S, Gong J et al. (2015) Genomic analysis of hybrid rice varieties reveals numerous superior alleles that contribute to heterosis. Nature communications 6 .

Li Y (2011) Some Recognitions of Shixia culture. Cultural Relics 5:48-54 (in Chinese).

Lu L, Zhao Z and Zheng Z (2005) The prehistoric and historic environments, vegetation and subsistence strategies at Sha Ha, Sai Kung, in AMO, Hong Kong. The ancient culture of Hong Kong: archaeological discoveries in Sha Ha, Sai Kung, 57-64.

Pang, H., Cai, H., Wang, X., 1995. Morphological classification research on the common wild rice (Oryza rufipogon Griff) in China. Acta Agronomica Sinica, 21(1), 17-24 (in Chinese).Xiang A (2005) Archaeological research on the prehistoric rice farming in Guangdong. Agricultural Archaeology 1: 149-155 (in Chinese).

Reimer PJ, Bard E, Bayliss A et al. (2013) IntCal13 and Marine13 radiocarbon age calibration curves 0-50,000 yearscal BP. Radiocarbon 55 :1869-1887

Silva F, Stevens CJ, Weisskopf A et al. (2015) Modelling the geographical origin of rice cultivation in Asia using the rice archaeological database. PLOS ONE 10: e0137024. 
Wang, X., Zhang, J., Chen, B., 1996. New discovery on the origin of rice agriculture in China. In: Wang X. and Sun C. (ed). The corpus of the origin and evolution of domesticated rice in China. China Agricultural University Press, Beijing, pp. 8-13 (in Chinese).

Yang X, Barton HJ, Wan Z et al. (2013) Sago-type palms were an important plant food prior to rice in southern subtropical China. PloS ONE 8:e63148.

Zhang C and Hung H (2010) The emergence of agriculture in southern China. Antiquity $84: 11-25$.

Zhang W, Xiang A, Qiu L et al. (2008) Studied on bi-peaked tubercles and signs of hull of ancient rice from the jiuwuhoushan site in the reaches of Xianghua river in Fengkai, Guangdong province. Chinese J Rice Sci 11: 103-106.

Zhao Z (2011) New archaeobotanical data for the study of the origins of agriculture in China. Current Anthropology 52: S295-2306.

You J, Zong W, Li X et al. (2013) The SNAC1-targeted gene OsSRO1c modulates stomatal closure and oxidative stress tolerance by regulating hydrogen peroxide in rice. Journal of experimental botany 64, 569-583.

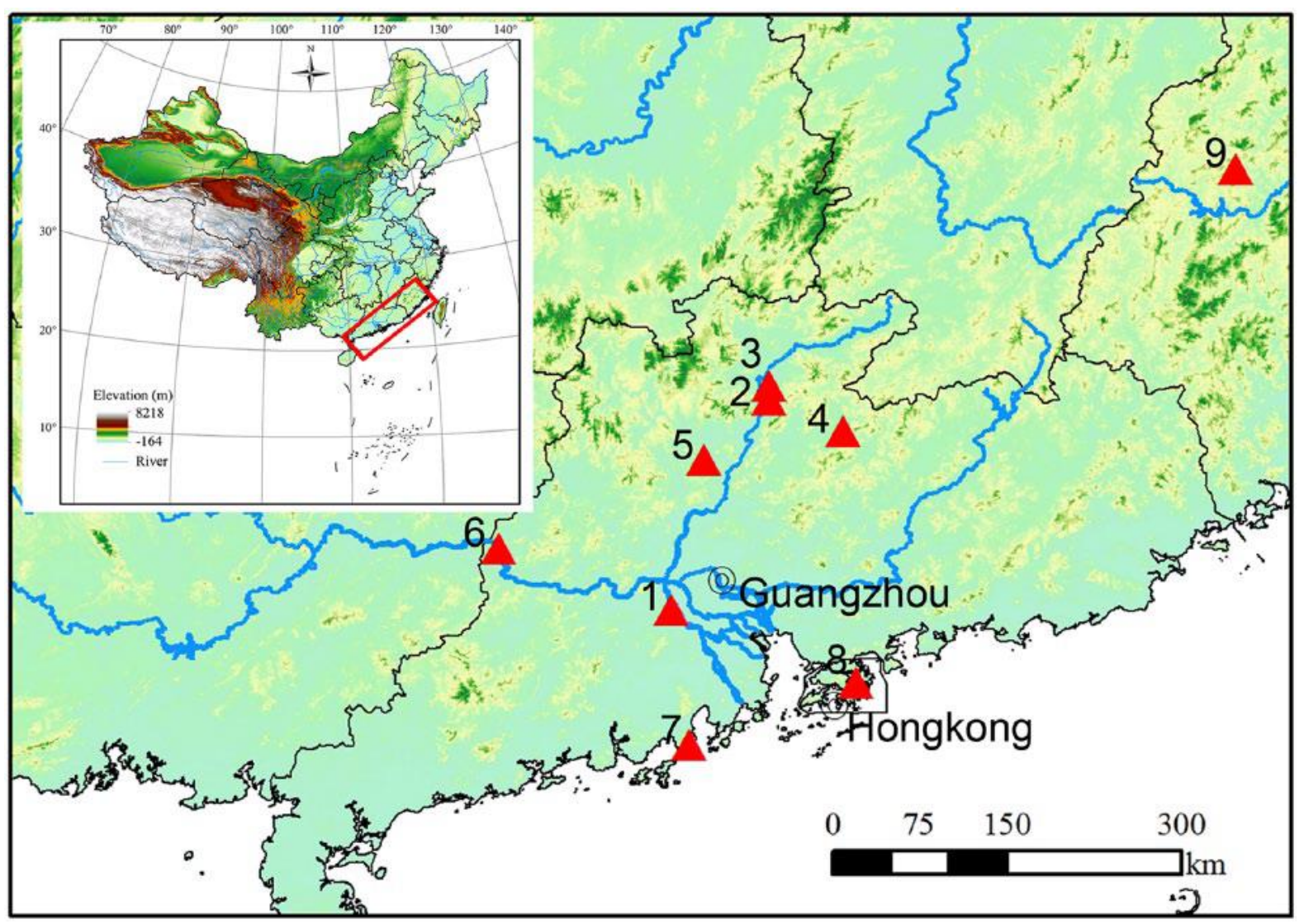


Figure 1. Location of archaeological sites for study and other sites mentioned in the text.

1, Guye; 2, Chuangbanyang; 3, Niling; 4, Xiajiaolong; 5, Shixia; 6,Guangxinhe; 7, Xincun; 8, Shaxia; 9, Nanshan

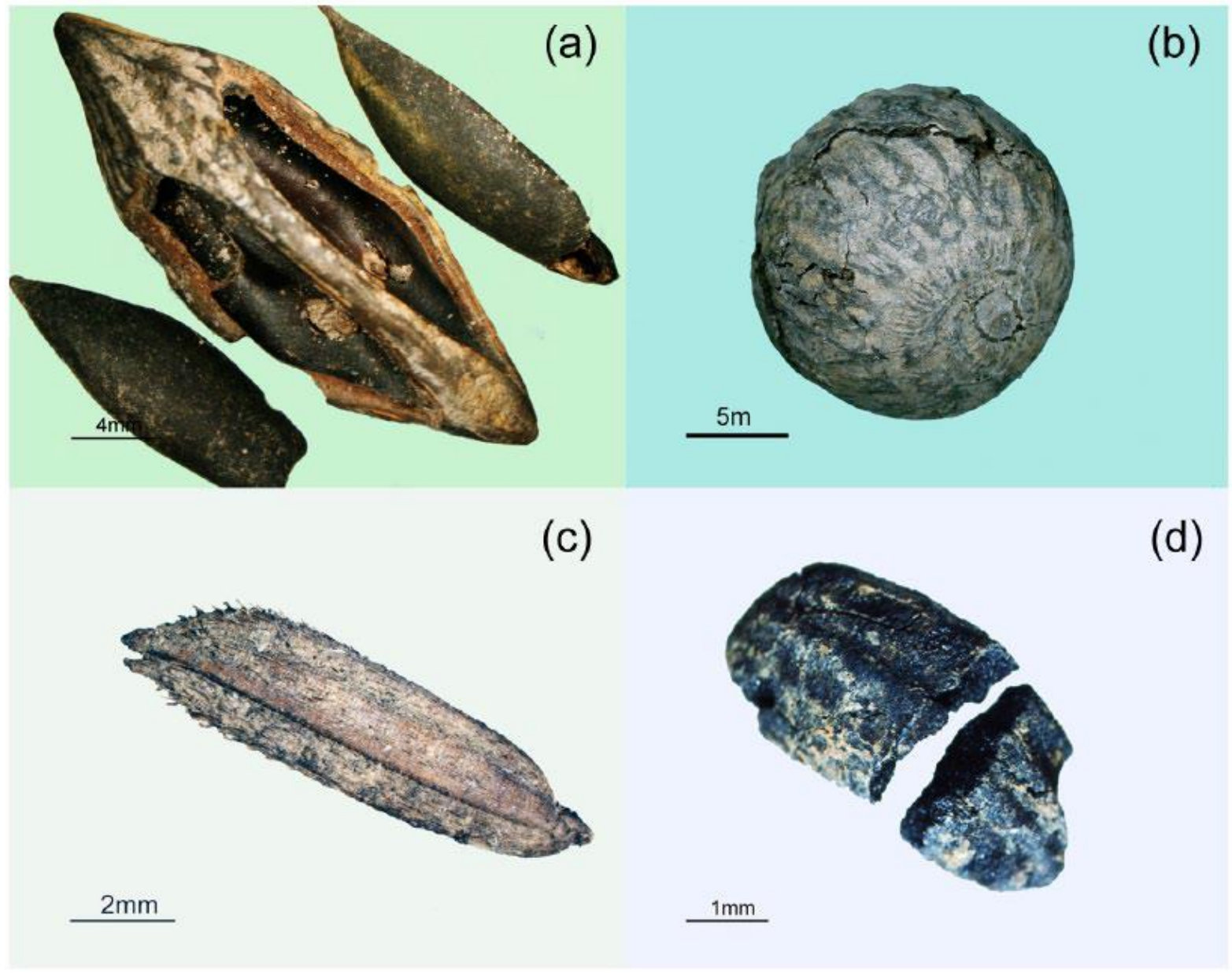

Figure 2. Charred plant remains for $\mathrm{AMS}{ }^{14} \mathrm{C}$ dating.

a, Chinese olives from the Guye site; b, an acorn from the Guye; c, a rice seed from the Guye; $d$, a charred rice seed from the Shixia. 
IntCall3 atmospheric auve (Reimer et al 2013)OxCal v3.10 Bronk Ramsey (2005); cub r:5 sd:12 prob usp[strat]

Sequence $\left\{A=78.1 \%\left(A^{\prime} c=60.0 \%\right)\right\}$

Boundary_Bound

Sequence Guangzhou

Phase Shixia 3

Beta-397662 99.6\%

$M$

Phase Shixia 2

BK75050 104.8\%

BK75046 $42.1 \%$

Phase Shixia 1

BK76024 116.1\%

Boundary Foraging/Farming

Phase Guye

BA111813 98.8\%

BA111819 96.9\%

BA111822 95.2\%

$1 \ldots$

BA111814 98.9\%

BA111817 86.3\%

BA111820 99.3\%

BA111823 99.9\%

BA111815 98.4\%

BA111818 99.0\%

BA111821 101.7\%

BA111824 98.4\%

Boundary Bound
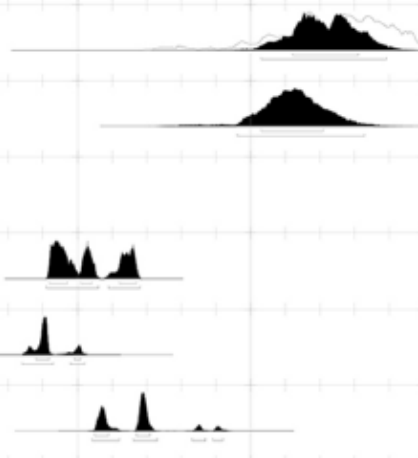

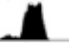

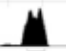

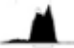

$M$

$1 d$

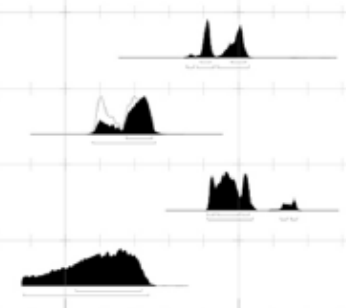

7500BP 7000BP 6500BP 6000BP 5500BP 5000BP 4500BP 4000BP $3500 \mathrm{BP}$

Calendar date

Figure3. Calibrated AMS ${ }^{14} \mathrm{C}$ dates from Shixia and Guye. IntCall3 atmospheric curve (Reimer et al. 2013); OxCal v3.10 Bronk Ramsey (2005). 


\begin{tabular}{llcc}
\hline Lab no. & Samples & Sample source & Calibrated/BC \\
\hline $\begin{array}{l}\text { Early Shixia Culture } \\
\text { BK76024 }\end{array}$ & Charcoal & M79 $/ \mathrm{BP}$ & $4220 \pm 110$ \\
$\begin{array}{l}\text { Middle Shixia Culture } \\
\text { BK75050 }\end{array}$ & Charcoal & M26 & $2730 \pm 155$ \\
BK75046 & Charcoal & M43 & $4020 \pm 100$ \\
\hline
\end{tabular}

Dates were cited from Guangdong Provincial Institute of Cultural Relics and Archaeology et al. (2014).

Table 1. Published dating data of the Shixia sites

\begin{tabular}{|c|c|c|c|c|c|c|}
\hline Lab no. & Samples & Field no. & Horizon & ${ }^{14} \mathrm{C}(\mathrm{BP})$ & Calibrated $(2 \sigma)$ & \\
\hline \multicolumn{7}{|l|}{ Guye site } \\
\hline \multirow[t]{3}{*}{ BAIII8I3 } & Soot & 06GGLTs09w08(5)-0I & Layer 5 & $4730 \pm 30$ & $3630 \mathrm{BC}(32.9 \%) 3580 \mathrm{BC}$ & $3640 \mathrm{BC}(64.7 \%) 3490 \mathrm{BC}$ \\
\hline & & & & & $3540 \mathrm{BC}(12.6 \%) 35 \mathrm{IO} \mathrm{BC}$ & $3460 \mathrm{BC}(30.7 \%) 3370 \mathrm{BC}$ \\
\hline & & & & & $3430 \mathrm{BC}(22.7 \%) 3380 \mathrm{BC}$ & \\
\hline \multirow[t]{3}{*}{ BAIII8I9 } & Cordia & 06GGLTs09w08(5)-C8-II & Layer 5 & $4850 \pm 30$ & $3700 \mathrm{BC}(4.5 \%) 3680 \mathrm{BC}$ & $3710 \mathrm{BC}(79.3 \%) 3630 \mathrm{BC}$ \\
\hline & & & & & $3660 \mathrm{BC}(56.9 \%) 3630 \mathrm{BC}$ & $3580 \mathrm{BC}(16.1 \%) 3530 \mathrm{BC}$ \\
\hline & & & & & $3550 \mathrm{BC}(6.8 \%) 3540 \mathrm{BC}$ & \\
\hline \multirow[t]{4}{*}{ BAIII 822} & Acorn & 06GGLTs09w08(5)-B3-I & Layer 5 & $4595 \pm 30$ & $3500 \mathrm{BC}(26.8 \%) 3460 \mathrm{BC}$ & $3500 \mathrm{BC}(37.2 \%) 3430 \mathrm{BC}$ \\
\hline & & & & & $3380 \mathrm{BC}(4 \mathrm{I} .4) 3340 \mathrm{BC}$ & $3380 \mathrm{BC}(47.8 \%) 3330 \mathrm{BC}$ \\
\hline & & & & & & $3220 \mathrm{BC}(5.7 \%) 3180 \mathrm{BC}$ \\
\hline & & & & & & $3160 \mathrm{BC}(4.7 \%) 3120 \mathrm{BC}$ \\
\hline BAIII8I4 & Soot & 06GGLTs I0w09(6-0I & Layer 6 & $4905 \pm 30$ & $3700 \mathrm{BC}(68.2 \%) 3650 \mathrm{BC}$ & $3760 \mathrm{BC}(95.4 \%) 3630 \mathrm{BC}$ \\
\hline BAIII8I7 & Chinese olive & 06GGLTs09w08(6-HI-I & Layer 6 & $4355 \pm 40$ & 3015 BC (68.2\%) 2910 BC & 3090 BC $(95.4 \%) 2890$ BC \\
\hline \multirow[t]{2}{*}{ BAIII 820} & Cordia & 06GGLTs09w08(6-PI-8 & Layer 6 & $4895 \pm 30$ & 3695 BC $(68.2 \%) 3645$ BC & $3760 \mathrm{BC}(1.4 \%) 3740 \mathrm{BC}$ \\
\hline & & & & & & $3720 \mathrm{BC}(94.0 \%) 3630 \mathrm{BC}$ \\
\hline BAIII823 & Acorn & 06GGLTs09w08(6)-J3-4 & Layer 6 & $4890 \pm 35$ & $3700 \mathrm{BC}(68.2 \%) 3645 \mathrm{BC}$ & $3770 \mathrm{BC}(95.4 \%) 3630 \mathrm{BC}$ \\
\hline \multirow[t]{3}{*}{ BAIII8I5 } & Soot & 06GGLTs 10 w08 $\odot-01$ & Layer 7 & $4715 \pm 30$ & $3630 \mathrm{BC}(18.4 \%) 3590 \mathrm{BC}$ & $3640 \mathrm{BC}(29.6 \%) 3560 \mathrm{BC}$ \\
\hline & & & & & $3530 \mathrm{BC}(15.9 \%) 3490 \mathrm{BC}$ & $3540 \mathrm{BC}(20.6 \%) 3490 \mathrm{BC}$ \\
\hline & & & & & $3430 \mathrm{BC}(34.0 \%) 3370 \mathrm{BC}$ & \\
\hline \multirow[t]{2}{*}{ BAIII8I8 } & Chinese olive & 06GGLTs09w08 $\oslash-A 3-1$ & Layer 7 & $4825 \pm 35$ & $3660 \mathrm{BC}(30.0 \%) 3630 \mathrm{BC}$ & $3700 \mathrm{BC}(39.1 \%) 3620 \mathrm{BC}$ \\
\hline & & & & & $3580 \mathrm{BC}(38.2 \%) 3530 \mathrm{BC}$ & $3610 \mathrm{BC}(56.3 \%) 3520 \mathrm{BC}$ \\
\hline \multirow[t]{2}{*}{ BAIII82I } & Cordia & 06GGLTs09w08@-FII-I & Layer 7 & $5095 \pm 30$ & $3960 \mathrm{BC}(17.2 \%) 3930 \mathrm{BC}$ & $3970 \mathrm{BC}(36.4 \%) 3890 \mathrm{BC}$ \\
\hline & & & & & $3880 \mathrm{BC}(51.0 \%) 3800 \mathrm{BC}$ & $3880 \mathrm{BC}(59.0 \%) 3790 \mathrm{BC}$ \\
\hline \multirow[t]{3}{*}{ BAIII824 } & Acorn & 06GGLTs09w08@-A3-2 & Layer 7 & $4755 \pm 25$ & $3640 \mathrm{BC}(9.5 \%) 3620 \mathrm{BC}$ & $3540 \mathrm{BC}(90.2 \%) 3510 \mathrm{BC}$ \\
\hline & & & & & 3610 BC $(44.2 \%) 3550$ BC & $3430 \mathrm{BC}(5.2 \%) 3380 \mathrm{BC}$ \\
\hline & & & & & $3540 \mathrm{BC}(14.5 \%) 3520 \mathrm{BC}$ & \\
\hline \multirow[t]{3}{*}{ BAIII 827} & Rice seed & 06GGLTS09W06@-Z2 & Layer 7 & $15 \pm 35$ & AD 1690 (12.9\%) AD 1730 & \\
\hline & & & & & AD $1810(48.9 \%)$ AD 1920 & \\
\hline & & & & & AD $1950(33.6 \%)$ AD 1960 & \\
\hline BAIII826 & Rice seed & 06GGLTS09W086)-Z14 & Layer 6 & $\begin{array}{l}\text { Modern } \\
\text { carbon }\end{array}$ & & \\
\hline \multirow[t]{3}{*}{ BAIII 825} & Rice seed & 06GGLTSIOW07(5)-Z10 & Layer 5 & $275 \pm 40$ & AD I 480 (87.9\%) AD I670 & \\
\hline & & & & & AD 1770 (6.1\%) AD 1800 & \\
\hline & & & & & AD 1940 (I.4\%) AD 1960 & \\
\hline \multicolumn{7}{|l|}{ Shixia } \\
\hline \multirow[t]{2}{*}{ Beta-397662 } & Rice seed & - & TomeM2I & $3810 \pm 30$ & $2340-2195 \mathrm{BC}$ & \\
\hline & & & & & $2175-2145 \mathrm{BC}$ & \\
\hline
\end{tabular}

Table 2. AMS ${ }^{14} \mathrm{C}$ dates of carbonized seeds from the sites of Guye and Shixia 